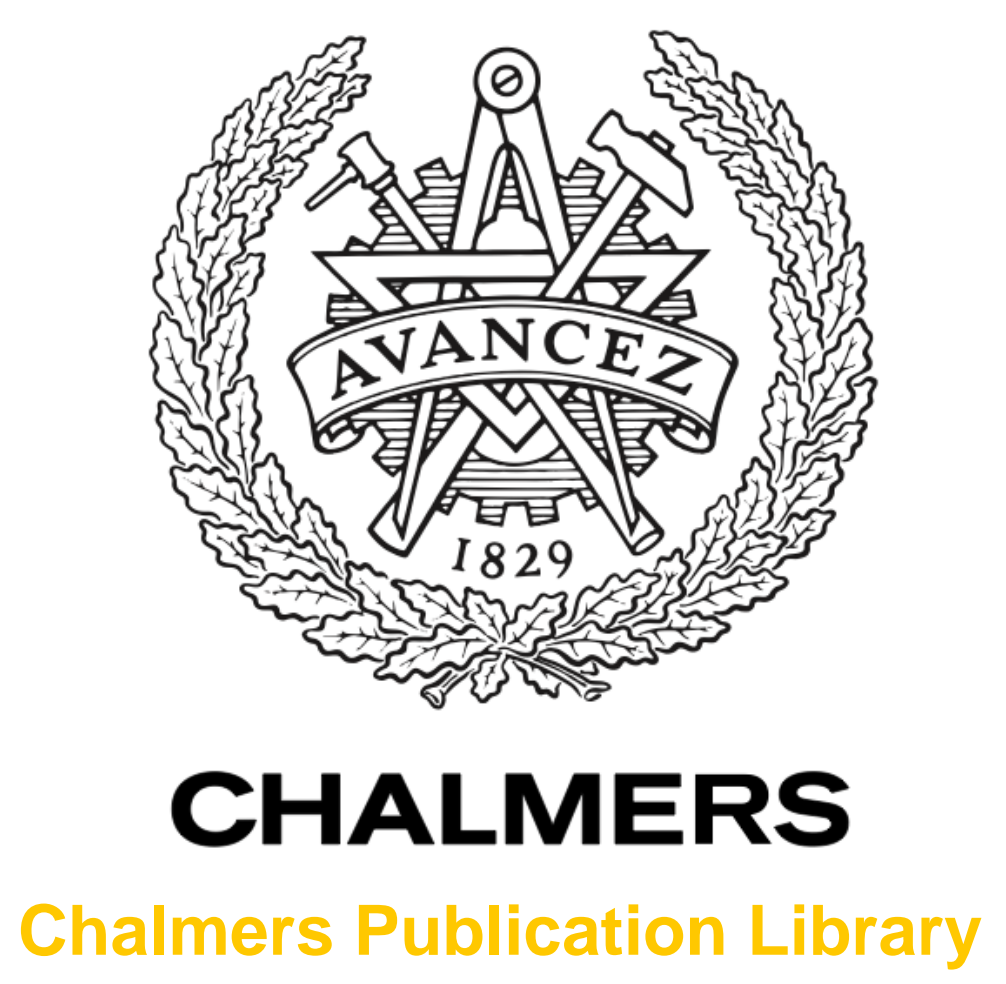

\title{
Galvanically split superconducting microwave resonators for introducing internal voltage bias
}

This document has been downloaded from Chalmers Publication Library (CPL). It is the author's version of a work that was accepted for publication in:

\section{Applied Physics Letters (ISSN: 0003-6951)}

Citation for the published paper:

de Graaf, S. ; Davidovikj, D. ; Adamyan, A. (2014) "Galvanically split superconducting microwave resonators for introducing internal voltage bias". Applied Physics Letters, vol.

104(5), pp. art no 052601.

http://dx.doi.org/10.1063/1.4863681

Downloaded from: http://publications.lib.chalmers.se/publication/196180

Notice: Changes introduced as a result of publishing processes such as copy-editing and formatting may not be reflected in this document. For a definitive version of this work, please refer to the published source. Please note that access to the published version might require a subscription. 


\title{
Galvanically split superconducting microwave resonators for introducing internal voltage bias
}

\author{
S. E. de Graaf, ${ }^{\text {a) }}$ D. Davidovikj, A. Adamyan, S. E. Kubatkin, and A. V. Danilov \\ Department of Microtechnology and Nanoscience, MC2, Chalmers University of Technology, \\ SE-41296 Gothenburg, Sweden
}

(Received 25 October 2013; accepted 15 January 2014; published online 3 February 2014)

\begin{abstract}
We present the design and performance of high-Q superconducting niobium nitride microwave resonators intended for use in hybrid quantum systems, coupling spin degrees of freedom to the cavity mode, both magnetically and electrically. We demonstrate a solution that allows to introduce static electric fields in the resonator without compromising the microwave performance. Quality factors above $10^{5}$ remain unchanged in strong applied static electric fields above $10 \mathrm{MV} / \mathrm{m}$ and magnetic fields up to $\sim 400 \mathrm{mT}$. By design, the configuration of the dc field matches that of the microwave field, especially advantageous for experiments on electrostatically controlled spin systems. (C) 2014 AIP Publishing LLC. [http://dx.doi.org/10.1063/1.4863681]
\end{abstract}

Superconducting thin-film resonators are widely used in experiments on Josephson-based qubits ${ }^{1-3}$ for their reduced mode volume and the ability to reach the strong coupling regime. While Josephson qubits are useful for quantum information processing, alternative methods are investigated to realize other functionalities such as quantum memories. Hybrid systems exploiting spin degrees of freedom are showing great potential for information storage ${ }^{4,5}$ and as coherent links between microwave and optical domains. ${ }^{6}$ The long relaxation times, especially of molecular compounds, ${ }^{7}$ could potentially increase the performance and diversity of these systems.

Recent advances in supra-molecular chemistry have revealed interesting spin systems with spin levels tuneable by electric fields. ${ }^{8,9}$ Systems of paramagnetic defects in piezoelectrics $^{10}$ and spin-orbit qubits ${ }^{11}$ can exhibit similar electrostatic spin tuning. This would allow for an efficient quantum memory with fast on-demand storage and retrieval of information by quick (and local) tuning of the spins in and out of resonance with the cavity. These interesting directions put a series of requirements on resonator design. In particular, the magnetic field required to bring these spin systems into resonance with the microwave field results in vortexinduced dissipation in the resonator. ${ }^{12,13}$ Another design challenge that we simultaneously focus on in this Letter is the possibility to introduce strong electric fields, without compromising microwave performance.

In a coplanar waveguide resonator (CPWR), the radiation losses are negligible as a result of a very small dipole moment in the structure: the microwave currents propagating in the center conductor are compensated for by almost equal currents propagating in the opposite direction in the nearby ground planes. In most cases, $Q_{\text {rad }} \gtrsim 10^{6}$ and scales with the ground-to-ground separation $s$ as $Q_{\text {rad }} \propto s^{-\alpha}$, ${ }^{14}$ where $\alpha=2$. In contrast, an unshielded strip $(s \rightarrow \infty)$ would act as an antenna with very large radiation losses. This also applies to small perturbations to the geometry of a coplanar transmission line (for example, $\alpha$ was found to increase to 2.3 for a meandered $\left.\mathrm{CPWR}^{14}\right)$.

a)Electronic mail: degraaf@chalmers.se
The introduction of a dc bias connection in the CPWR geometry constitutes a radical change to the design. It significantly disrupts the ground plane currents, leading to large radiation losses. This situation can be improved by careful engineering and matching of impedances in the circuit, either by high impedance inputs ${ }^{15-17}$ or sophisticated band-stop filter engineering. ${ }^{18}$ While the former method has been demonstrated for quality factors on the order of a few thousands, high quality filter matching can potentially give much lower loss. ${ }^{18}$ However, this method becomes unpractical in strong magnetic fields, since the resonator and filter properties will change differently (due to kinetic inductance), and matching conditions are no longer the same.

For the design presented here, we solve the issue of introducing dc bias by instead creating a resonator that has a very small dipole moment, i.e., the currents flowing in the ground planes become negligible. This allows us to split the ground plane encompassing the resonator without compromising the microwave performance and to use the two halfplanes for dc biasing.

The resonator design can be derived from merging two identical $\lambda / 4$ resonators (shown in Fig. 1(a)). Each of the two basic units is similar to the design originally presented in Ref. 19. To achieve good magnetic field performance, we load the two parallel strips of the resonator with a dense capacitance, acting as a metamaterial that reduces the phase velocity in the resonator. One advantage is that this minimises magnetic field induced dissipation by distributing microwave currents throughout the structure. The lower phase velocity results in a lower impedance $(\approx 30 \Omega)$ which further decouples the resonance mode from free space radiation. Additionally, the symmetry and the dense network of parallel leads within the resonator, each carrying a current in the opposite direction as its neighboring leads, ensure that the microwave currents create dipole moments which cancel each other. With mainly quadrupole terms left unbalanced, the encircling ground plane currents become small.

In the next step (Fig. 1(b)), we arrive at two weakly coupled resonators. Such a system has two eigenmodes: symmetric and antisymmetric. Here, the currents which go 
(a)

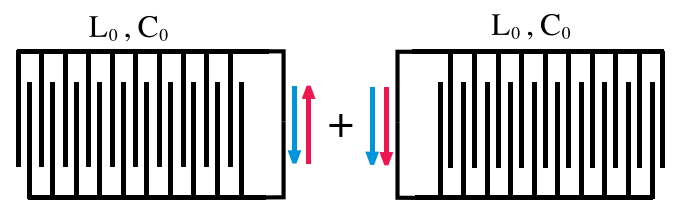

(b)

(c)

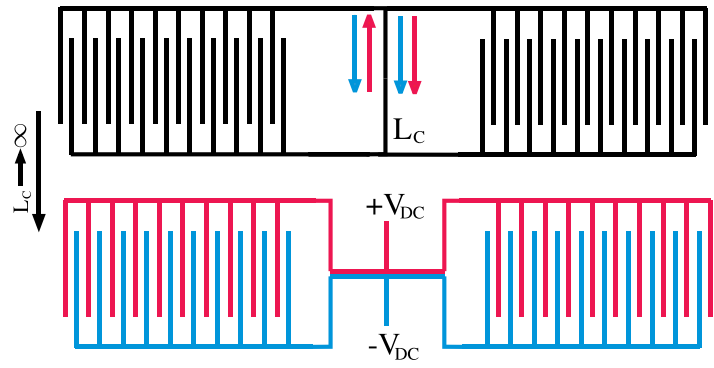

(d)

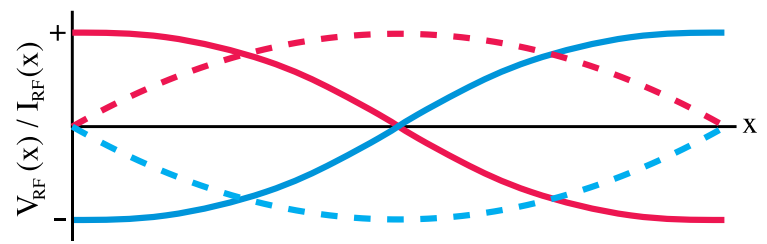

FIG. 1. (a) Two identical coupled resonators. Red (symmetric) and blue (antisymmetric) arrows indicate direction of currents for the two coupled modes. (b) Same resonators coupled via the common inductance $L_{C}$. (c) By removing the common link $\left(L_{C} \rightarrow \infty\right)$, we arrive at the final structure used in this work. It only has the antisymmetric mode, and it can easily be internally dc biased. (d) Microwave voltage (solid) and current (dash) along the resonant structure in (c) for the two segments.

through the inductive link $L_{c}$ sum up for the symmetric mode $\left(\omega_{s}=1 / \sqrt{C_{0}\left(L_{0}+2 L_{c}\right)}\right)$ and cancel each other for the antisymmetric mode $\left(\omega_{a}=1 / \sqrt{C_{0} L_{0}}\right)$, so that if $L_{c} \rightarrow \infty$ the symmetric mode vanishes and the anti-symmetric mode stays at its original frequency. The final design (Fig. 1(c)) is a balanced $\lambda / 2$ resonator with a current and voltage distribution as shown in Fig. 1(d).

Alternatively, the resonator in Fig. 1(c) can be described as a coplanar stripline resonator loaded with a dense distributed capacitance. We keep parts of this coplanar stripline geometry in the center of the resonator (voltage node), since it is more advantageous for coupling to small spin ensembles. Microwave currents passing through the two central segments have opposite directions, so that inbetween the two segments the $\mathrm{z}$ component of the magnetic field is enhanced. The detailed structure of the magnetic microwave field and the electric field in this area is shown in Fig. 2(a). The coplanar stripline section also has a higher propagation velocity than the capacitively loaded counterpart which spatially delocalizes the voltage node in the resonator, advantageous for connecting dc bias lines. One section of the resonator is capacitively coupled to a transmission line $\left(C_{c}\right)$ and to maintain symmetry we couple the other section to the ground via an identical capacitance.

Due to the well-balanced design, the ground plane can be split into two parts, as shown in Fig. 2(b). To make the slot between the two half-planes even more transparent for residual currents, the capacitor linking two halves is also implemented as a dense interdigitated structure. Furthermore, the splitground design provides for a path for flux to escape from around the resonator, further improving its magnetic field performance, eliminating low-field hysteretic effects, and reducing flux focusing. ${ }^{12,19,20}$ (a)

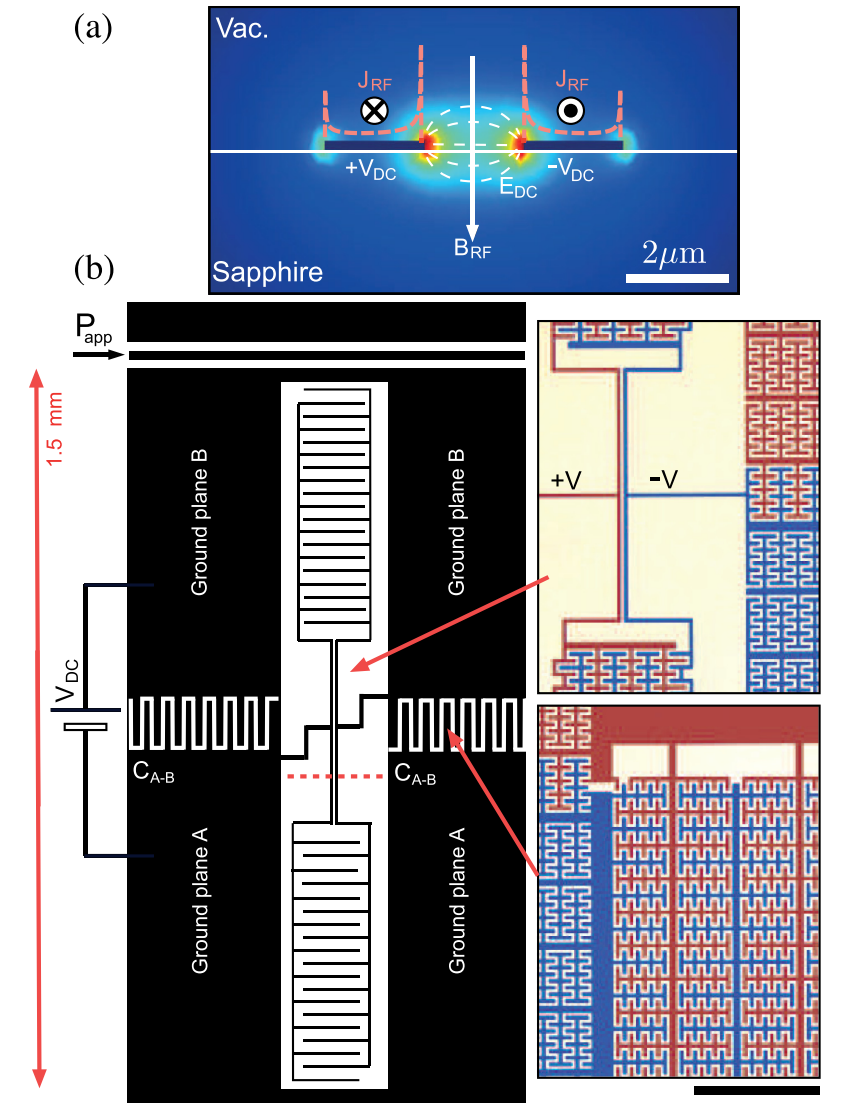

FIG. 2. (a) Cross-section near the current maximum (dashed line in (b)). Color gradient shows microwave magnetic field intensity, obtained with COMSOL simulations, and the dashed red line shows the normalized microwave current density across the strip. (b) Left: Schematic sketch of the resonator and ground plane design. Right: False color optical image taken near the current node of the resonator (top) and the high density ground capacitor (bottom). Red and blue areas indicate the two galvanically isolated segments of superconductor. Scale bar is $60 \mu \mathrm{m}$.

Finally, the low dissipation dc bias connection is supplied to the two resonator segments via links coupled to the voltage nodes (here, we use the geometric center of the resonator), as illustrated in Fig. 2(b). Due to the coupling capacitance, we expect a small shift of the voltage node. This shift can be roughly estimated from $\delta x=\lambda C_{c} / C_{0} \approx 0.0025 \lambda<10 \mu \mathrm{m}$. The expected radiation loss from placing the dc bias connections at the geometric center of the resonator therefore gives $Q_{\mathrm{dc}} \approx 10^{5} .{ }^{21}$

The resonators were made from niobium nitride $(\mathrm{NbN})$, $140 \mathrm{~nm}$ thick, sputtered onto a C-plane sapphire substrate and patterned using electron beam lithography and reactive ion etching in a $\mathrm{NF}_{3}$ plasma. We chose $\mathrm{NbN}$ for its high thermodynamic critical field $\left(H_{c}\right)$ and sapphire substrates since they have a high dielectric breakdown field. Apart from this, the resonators inherit the same properties as previously demonstrated for similar $\mathrm{Nb}$ resonators. ${ }^{19}$ The measurements shown in Fig. 3(a) were performed in a helium flow cryostat at $1.8 \mathrm{~K}$ using a vector network analyzer and the measured transmission signal $\left(S_{21}\right.$, see inset Fig. 3(a)) was fitted using a standard method for microwave resonators ${ }^{22}$ to extract internal $\left(Q_{\text {int }}\right)$ and coupling $\left(Q_{c}\right)$ quality factors. We also separate the dissipation induced by magnetic fields from other loss mechanisms by defining the field-induced loss tangent $Q_{B}^{-1}=Q_{\text {int }}^{-1}(B)-Q_{\text {int }}^{-1}(B=0),{ }^{13}$ which is the quantity shown 
(a)
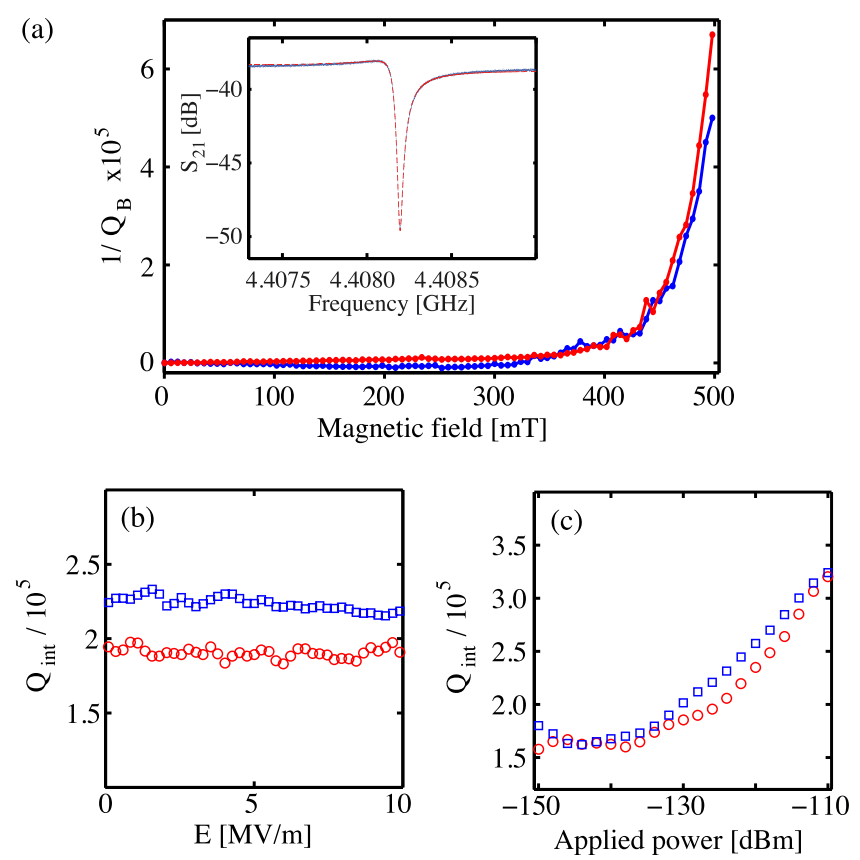

FIG. 3. (a) Parallel magnetic field induced dissipation for two typical resonators (different colors). Inset: Measured (dots) resonance lineshape and fit (dashed line) at zero applied static electric and magnetic field. The coupling of the two resonators shown here was extracted to $Q_{c}=71000$ and 57000 $(1.8 \mathrm{~K})$ and the probing power was $-70 \mathrm{dBm}$. (b) Dependence of the internal quality factor versus applied static electric field for the same resonators measured at $300 \mathrm{mK}$ and near single photon population of the resonators $(-125 \mathrm{dBm}$ excitation, $B=0)$. (c) Power dependence of the internal Q measured at $300 \mathrm{mK}(B=0, E=0)$.

in Fig. 3(a). For the measurements in magnetic field, we do not perform any tedious alignment of the field to make it completely parallel with the $\mathrm{NbN}$ film, instead we rely on the exceptionally low flux focusing factor ${ }^{20}(\sim 2-3)$ of our design. The data at low powers (around single photon population of the resonators) in Figs. 3(b) and 3(c) were obtained in a different cryostat with a base temperature of $300 \mathrm{mK}$. The magnetic field available in this cryostat $(400 \mathrm{mT})$ was not sufficient to noticeably affect the resonators at all, which is why we instead present high field data taken at $1.8 \mathrm{~K}$.

The data in Fig. 3(a) show that the magnetic field induced losses are low enough not to degrade the $\mathrm{Q}$ of the resonator on the level of $10^{5}$ up to $400 \mathrm{mT}$, and these losses are expected to have a very weak temperature and power dependence. ${ }^{19}$ No hysteresis is observed for the measured fields in Fig. 3(a) (i.e., the quality factors remain the same when returning to $B=0$ ); however, exposing the resonator to even stronger fields will eventually lead to a hysteretic behavior due to trapped flux in the structure.

In Fig. 3(b), we show that applied electric fields of up to $10 \mathrm{MV} / \mathrm{m}$ have no observable impact on the quality factor even for very low excitation powers. Furthermore, the quality factors saturate at a level of $Q_{\text {int }}=1.3-2 \cdot 10^{5}$ (observed spread over a total of eight different resonators) below single photon population (see Fig. 3(c)). This is in accordance with the expected dielectric loss tangent in our sapphire substrates.

It is possible to qualitatively explain these high quality factors by considering different ground-plane geometries. We have seen ${ }^{19}$ that the initially high $Q_{\text {int }}$ in the presence of ground planes drops to $Q_{\text {int }} \approx 3-4 \cdot 10^{4}$ when the ground planes are removed. This suggests that the residual dipole moment in the presented design is up to 40000 times less than the dipole moment of an unshielded CPWR, and ground plane currents are reduced by the same amount. Still, splitting the ground plane in two parts results in the delocalization of the circulating currents and dissipation. However, in our design, the capacitance per unit area is very large, and this ensures that the circulating currents are localized on a length-scale much smaller than the wavelength, $\lambda$. This is another key requirement for maintaining a high $\mathrm{Q}$ resonance. It also allows us to further relax the requirement of connecting the bias to the resonator exactly at its voltage node and still maintain a high $\mathrm{Q}$, and it partially explains why the measured $Q_{\text {int }}$ is even larger than the expected value of $Q_{\mathrm{dc}} \approx 10^{5}$. The inductance of the dc bias lines also contributes to further increase $Q_{\mathrm{dc}}$. Additionally, any resistive elements (such as bonding wires for supplying dc) in this design are placed far from any currents around the resonant structure and can be neglected as a source of dissipation.

Finally, we also note that the presented design can easily be extended to also support current dc-bias by operating it as a $3 \lambda / 2$ resonator and introducing another dc connection at the second voltage node.

In summary, we have demonstrated a split-ground plane superconducting resonator design in which we internally introduced dc bias to the resonator. Our measurements show that this does not significantly contribute to resonator loss on the level of $Q=10^{5}$. Furthermore, the presented design is compatible with current technology of making the resonator resilient to magnetic field, and our $\mathrm{NbN}$ resonators show quality factors above $10^{5}$, unaffected by applied parallel field up to $\sim 400 \mathrm{mT}$, making these resonators good candidates for coupling to, for example, electrostatically tuneable spin ensembles.

We acknowledge EU FP7 programme under the grant agreement "ELFOS," the Marie Curie Initial Training Action (ITN) Q-NET 264034, the Swedish Research Council $\mathrm{VR}$, and the Linnaeus centre for quantum engineering for financial support.

${ }^{1}$ A. Wallraff, D. I. Schuster, A. Blais, L. Frunzio, R.-S. Huang, J. Majer, S. Kumar, S. M. Girvin, and R. J. Schoelkopf, Nature (London) 431, 162 (2004).

${ }^{2}$ G. Wendin and V. Shumeiko, Low Temp. Phys. 33, 724 (2007).

${ }^{3}$ I. Siddiqi, Supercond. Sci. Technol. 24, 091002 (2011).

${ }^{4}$ Y. Kubo, F. R. Ong, P. Bertet, D. Vion, V. Jaques, D. Zheng, A. Dréau, J. F. Roch, A. Auffeves, F. Jelezeko, J. Wrachtrup, M. F. Barthe, P. Bergonzo, and D. Esteve, Phys. Rev. Lett. 105, 140502 (2010).

${ }^{5}$ D. I. Schuster, A. P. Sears, E. Ginossar, L. DiCarlo, L. Frunzio, J. J. L. Morton, H. Wu, G. A. D. Briggs, B. B. Buckley, D. D. Awschalom, and R. J. Schoelkopf, Phys. Rev. Lett. 105, 140501 (2010).

${ }^{6}$ P. Rabl, D. DeMille, J. M. Doyle, M. D. Lukin, R. J. Schoelkopf, and P. Zoller, Phys. Rev. Lett. 97, 033003 (2006).

${ }^{7}$ L. Bogani and W. Wernsdorfer, Nature Mater. 7, 179 (2008).

${ }^{8}$ J. Lehmann, A. Gaita-Ariño, E. Coronado, and D. Loss, J. Mater. Chem. 19, 1672 (2009).

${ }^{9}$ M. Trif, F. Troiani, D. Stepanenko, and D. Loss, Phys. Rev. Lett. 101, 217201 (2008).

${ }^{10}$ R. E. George, J. P. Edwards, and A. Ardavan, Phys. Rev. Lett. 110, 027601 (2013).

${ }^{11}$ S. Nadj-Perge, V. S. Pribiag, J. W. G. van den Berg, K. Zuo, S. R. Plissard, E. P. A. M. Bakkers, S. M. Frolov, and L. P. Kouwenhoven, Phys. Rev. Lett. 108, 166801 (2012). 
${ }^{12}$ D. Bothner, T. Gaber, M. Kemmler, D. Koelle, and R. Kleiner, Phys. Rev. B 86, 014517 (2012).

${ }^{13}$ C. Song, T. W. Heitmann, M. P. DeFeo, K. Yu, R. McDemott, M. Neeley, J. M. Martinis, and B. L. T. Plourde, Phys. Rev. B 79, 174512 (2009).

${ }^{14}$ J. M. Sage, V. Bolkhovsky, W. D. Oliver, B. Turek, and P. B. Welander, J. Appl. Phys. 109, 063915 (2011).

${ }^{15}$ R. N. Simons, Coplanar Waveguide Circuits, Components, and Systems (Wiley, New York, 2001), and references therein.

${ }^{16}$ F. Chen, A. J. Sirois, R. W. Simmonds, and A. J. Rimberg, Appl. Phys. Lett. 98, 132509 (2011).

${ }^{17}$ K. D. Petersson, L. W. McFaul, M. D. Schroer, M. Jung, J. M. Taylor, A. A. Houck, and J. R. Petta, Nature (London) 490, 380 (2012).

${ }^{18}$ S.-X. Li and J. B. Kycia, Appl. Phys. Lett. 102, 242601 (2013).
${ }^{19}$ S. E. de Graaf, A. V. Danilov, A. Adamyan, T. Bauch, and S. E. Kubatkin, J. Appl. Phys. 112, 123905 (2012).

${ }^{20}$ J. E. Healey, T. Lindström, M. S. Colclough, C. M. Muirhead, and A. Ya. Tzalenchuk, Appl. Phys. Lett. 93, 043513 (2008).

${ }^{21}$ We note that displacing any dc bias connection from the true voltage node by an amount $\delta x$ will result in a dissipation $Q_{\mathrm{dc}}^{-1}=2(\delta x / \lambda)^{2}=2\left(C_{c} / C_{0}\right)^{2}$. This is a very simplified case that does not take into account ground plane radiation and the impedance of the dc links. Due to the reduced propagation velocity, we have $\lambda / 2 \approx 1.5 \mathrm{~mm}$ for $f_{0}=4 \mathrm{GHz}$. The contribution from the coplanar strip region near the voltage nodes can be neglected, since it constitutes $\sim 1 \%$ of $C_{0}$.

${ }^{22}$ M. S. Khalil, M. J. A. Stoutimore, F. C. Wellstood, and K. D. Osborn, J. Appl. Phys. 111, 054510 (2012). 


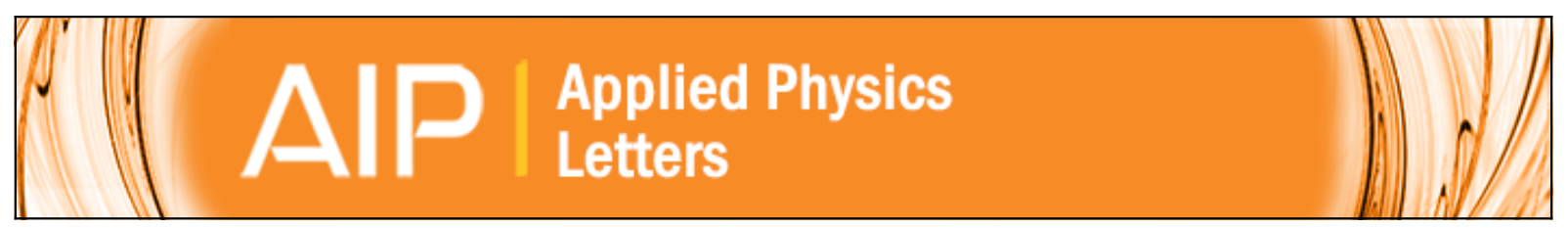

\section{Galvanically split superconducting microwave resonators for introducing internal voltage bias}

S. E. de Graaf, D. Davidovikj, A. Adamyan, S. E. Kubatkin, and A. V. Danilov

Citation: Applied Physics Letters 104, 052601 (2014); doi: 10.1063/1.4863681

View online: http://dx.doi.org/10.1063/1.4863681

View Table of Contents: http://scitation.aip.org/content/aip/journal/apl/104/5?ver=pdfcov

Published by the AIP Publishing

\section{Articles you may be interested in}

Superconducting coplanar waveguide resonators for low temperature pulsed electron spin resonance spectroscopy

Rev. Sci. Instrum. 84, 025116 (2013); 10.1063/1.4792205

An analysis method for asymmetric resonator transmission applied to superconducting devices

J. Appl. Phys. 111, 054510 (2012); 10.1063/1.3692073

Reducing microwave loss in superconducting resonators due to trapped vortices

Appl. Phys. Lett. 95, 232501 (2009); 10.1063/1.3271523

Intermodulation gain in nonlinear $\mathrm{NbN}$ superconducting microwave resonators

Appl. Phys. Lett. 88, 022508 (2006); 10.1063/1.2164925

Performance of superconducting microwave devices passivated with dielectric materials

Appl. Phys. Lett. 71, 1564 (1997); 10.1063/1.119966

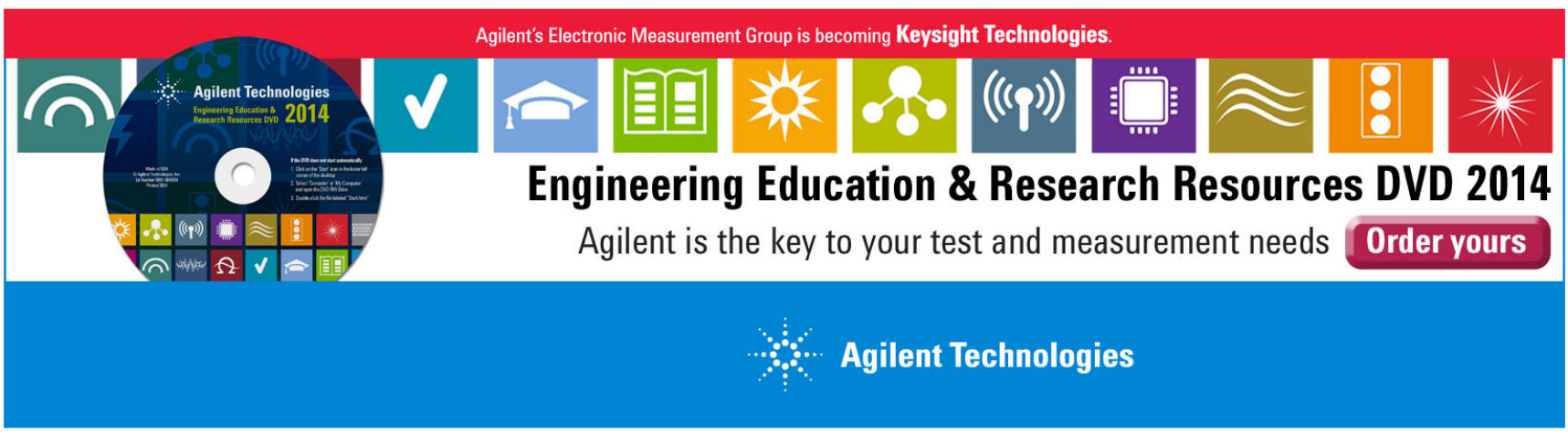

